\title{
Morsures de chien: annoncez les cas de blessure!
}

Office vétérinaire fédéral OVF

Base légale de l'obligation d'annoncer: Ordonnance sur la protection des animaux (en vigueur depuis le 1.9.2008), Art. 78 Annonce des accidents 1 Les vétérinaires, les médecins, les responsables de refuges ou de pensions pour animaux, les éducateurs canins et les organes des douanes sont tenus d'annoncer au service cantonal compétent: a. les accidents causés par un chien qui a gravement blessé un être humain ou un animal, et

b. les chiens qui présentent un comportement d'agression supérieur à la norme.

2 Les cantons peuvent soumettre d'autres catégories de personnes à l'obligation d'annoncer.

Correspondance: Regula Kennel

Office vétérinaire fédéral OVF

Communication

Schwarzenburgstrasse 155

CH-3003 Bern-Liebefeld

Tél. 0313238496

Fax 0313238570

regula.kennel[at]bvet.admin.ch
L'annonce des blessures causées par des morsures de chien, que la victime soit humaine ou animale, est obligatoire dans notre pays depuis 2006. Tout médecin, tout vétérinaire qui traite une blessure due à la morsure d'un chien doit déclarer l'accident à l'office vétérinaire de son canton. De même, tout éducateur canin ou tout vétérinaire doit signaler les chiens dont le comportement est suspect.

Ce système qui est en place mérite d'être encore amélioré: on est encore très loin d'une annonce systématique de tous les accidents par morsure et les données recueillies restent lacunaires. Il est donc important que les médecins et les vétérinaires soient conscients de leurs devoirs à cet égard et sachent comment procéder. En annonçant les cas, les professionnels de la santé humaine et animale peuvent contribuer de manière déterminante à la prévention des accidents causés par les morsures de chien.

En principe, lorsqu'un chien a gravement blessé un être humain ou un animal ou lorsqu'il présente un comportement d'agression supérieur à la norme, l'office vétérinaire cantonal doit être informé. Mais que signifie exactement «a gravement blessé» et que faut-il entendre par «un comportement d'agression supérieur à la norme»?

Selon les directives techniques de l'Office vétérinaire fédéral OVF en la matière, une blessure est considérée comme «grave» lorsque la victime, humaine ou animale, doit être prise en charge par un médecin, respectivement un vétérinaire. En d'autres termes, les médecins et les vétérinaires doivent déclarer tous les cas où ils sont consultés pour traiter une blessure due à la morsure d'un chien. Que la victime soit une personne ou un chien ne fait pas de différence. Cette obligation d'annoncer a une fonction préventive: elle doit servir à détecter le plus tôt possible les chiens dont le comportement suscite des inquiétudes. L'identité du patient ou du propriétaire d'un chien blessé ne peut être communiquée par le médecin, respectivement le vétérinaire qu'avec le consentement exprès de ces personnes et les annonces anonymes sont admises.

Quant à l'obligation d'annoncer les chiens qui présentent «un comportement d'agression supérieur à la norme», elle pose des problèmes d'application plus difficiles. La notion ainsi visée, à savoir les modes de comportement qui représentent un danger pour l'être humain ou l'animal, et sujette à interprétation. L'obligation d'annoncer de tels comportements est connue des vétérinaires et des médecins, des éducateurs canins et des responsables de refuges pour animaux, mais, là encore, le nombre de cas an- noncés est sans doute nettement inférieur au nombre de cas où il y a danger réel. En l'occurrence il serait donc judicieux d'appliquer le principe: «Mieux vaut une fois de trop qu'une fois de pas assez!»

\section{Que se passe-t-il lorsqu'un cas a été annoncé?}

Chaque fois qu'un cas lui est annoncé, le service vétérinaire cantonal compétent doit faire une enquête pour déterminer et évaluer la dangerosité du chien. Dans la moitié des cas environ, aucune mesure ne s'impose; dans un tiers des cas, un risque particulier est identifié. Généralement, le service vétérinaire cantonal donne un avertissement au détenteur et l'enjoint à respecter son devoir de surveillance. Dans les cas graves, d'autres mesures, des plus légères aux plus contraignantes, peuvent être prises: laisse obligatoire, port obligatoire de la muselière, remise du chien à un autre propriétaire, interdiction faite au propriétaire de détenir un chien, voire mise à mort du chien si la sécurité ne peut être assurée autrement.

\section{Ce qu'il faut encore savoir}

Les formulaires d'annonce sont disponibles auprès des services vétérinaires cantonaux et peuvent être téléchargés sur leur site Internet. Vous trouverez les liens vers les services vétérinaires cantonaux sur la page Internet de l'OVF: www.ovf.admin.ch $\rightarrow$ Thèmes $\rightarrow$ Service vétérinaire suisse $\rightarrow$ Services vétérinaires cantonaux. Il est recommandé aux médecins et aux vétérinaires d'avoir quelques formulaires en réserve. Le service vétérinaire compétent est toujours celui du canton où la blessure a été traitée ou là où le comportement du chien a attiré l'attention.

\section{Données factuelles et chiffrées}

Au total, 5090 cas impliquant des chiens ont été annoncés en 2009 , ce qui représente une augmentation de 10 pour cent par rapport à l'année précédente. Dans plus de la moitié des cas d'accidents par morsure, le chien est connu de la victime. Les enfants sont particulièrement exposés. Ils sont plus souvent victimes d'accidents par morsures de chien et leurs blessures sont plus graves. Plus de la moitié d'entre eux sont mordus au cou et à la tête, ce qui est plus rare chez les adultes. Les chiens de petite taille sont responsables de morsures chez un quart des enfants. Les chiens de taille moyenne et les chiens de grande taille sont responsables chacun d'un tiers des accidents par morsure.

Les données fournies sur les types de chiens impliqués étant très hétérogènes, il est très difficile de faire une statistique fiable en fonction des races. Les chiens les plus représentés sont les bergers et les bouviers, à savoir les chiens les plus appréciés et les plus fréquemment rencontrés en Suisse.

(Source: statistique des morsures de chien 2009. Edité par l'Office vétérinaire fédéral OVF et par l'Association suisse des vétérinaires cantonaux.) 